\title{
Leitthema
}

Gefässchirurgie 2016 · [Suppl 2]: 21:S37-S44 DOI 10.1007/s00772-016-0156-4

Published online: 12 July 2016

(c) The Author(s) 2016. This article is available at SpringerLink with Open Access

CrossMark

H. Jalaie ${ }^{1}$ K. Schleimer ${ }^{1}$ M. E. Barbati' $\cdot$ A. Gombert ${ }^{1} \cdot$ J. Grommes ${ }^{1}$ M. A. F. de Wolf $^{2} \cdot$ R. de Graaf $\cdot$ C. H. A. Wittens ${ }^{1,2}$

${ }^{1}$ Klinik für Gefäßchirurgie, Universitätsklinikum der RWTH Aachen, Europäisches Gefäßzentrum AachenMaastricht, Aachen, Germany

${ }^{2}$ European Vascular Center Aachen-Maastricht, Maastricht University Medical Center, Maastricht, The Netherlands

\section{Interventional treatment of postthrombotic syndrome}

currence of PTS significantly reduces the quality of life [21] and has considerable socioeconomic consequences [18]. A total of six different PTS scoring systems and classifications have been established, which assign a points score to the various symptoms and findings. Depending on this points score, PTS is classified as mild, moderate or severe. The Villalta score is the most commonly used system for diagnosis and classification (- Table 1). The Brandjes and Ginsberg scores are also used only for PTS. The Widmer score, the CEAP classification ( $\mathrm{C}=$ clinical condition, $\mathrm{E}=$ etiology, $\mathrm{A}=$ anatomical location, $\mathrm{P}=$ pathophysiology) and the venous clinical severity score (VCSS) are used for the classification of all chronic venous diseases [36].

\section{Pathogenesis}

Postthrombotic obstruction involving increased outflow resistance, damaged venous valves and reflux as well as fibrotic venous walls with reduced compliance cause venous hypertension [8, $13,28]$. The increased venous pressure is transmitted to the capillaries, which then dilate and increased capillary permeability also occurs. This results in edema, inflammation and pigmentation due to hemosiderin deposits and, thus, ultimately to the typical skin changes and venous ulcerations seen in PTS. Venous hypertension also causes distension of the deep veins and increased valve insufficiency, which is also transmitted to the superficial venous system via the perforating veins (secondary varicosis) $[12,16]$. According to current knowl- edge, proximal stenosis or occlusion contributes more to the development of venous hypertension and PTS than reflux [19, 20, 33, 35].

\section{Conservative treatment}

Compression therapy and anticoagulation form the basis for the conservative management of PTS. Overall, compression therapy reduces venous pressure,

Table 1 Criteria used in the Villalta score

\begin{tabular}{|c|c|c|c|c|}
\hline & None & Mild & $\begin{array}{l}\text { Mod- } \\
\text { erate }\end{array}$ & Severe \\
\hline \multicolumn{5}{|c|}{ Subjective symptoms } \\
\hline Pain & 0 & 1 & 2 & 3 \\
\hline Cramps & 0 & 1 & 2 & 3 \\
\hline $\begin{array}{l}\text { Leg heavi- } \\
\text { ness }\end{array}$ & 0 & 1 & 2 & 3 \\
\hline Parasthesia & 0 & 1 & 2 & 3 \\
\hline Pruritus & 0 & 1 & 2 & 3 \\
\hline \multicolumn{5}{|l|}{ Clinical signs } \\
\hline $\begin{array}{l}\text { Pretibial } \\
\text { edema }\end{array}$ & 0 & 1 & 2 & 3 \\
\hline $\begin{array}{l}\text { Skin in- } \\
\text { duration }\end{array}$ & 0 & 1 & 2 & 3 \\
\hline $\begin{array}{l}\text { Hyperpig- } \\
\text { mentation }\end{array}$ & 0 & 1 & 2 & 3 \\
\hline Erythema & 0 & 1 & 2 & 3 \\
\hline $\begin{array}{l}\text { Venous } \\
\text { ectasia }\end{array}$ & 0 & 1 & 2 & 3 \\
\hline $\begin{array}{l}\text { Pain on } \\
\text { calf com- } \\
\text { pression }\end{array}$ & 0 & 1 & 2 & 3 \\
\hline $\begin{array}{l}\text { Venous } \\
\text { ulcer }\end{array}$ & $\begin{array}{l}\text { Not } \\
\text { presen }\end{array}$ & Preser & & \\
\hline
\end{tabular}

Evaluation result 0-4 no postthrombotic syndrome (PTS), 5-9 mild PTS, 10-14 moderate PTS, $15-33$ severe PTS
The German version of this article can be found under doi:10.1007/s00772-015-0092-8 


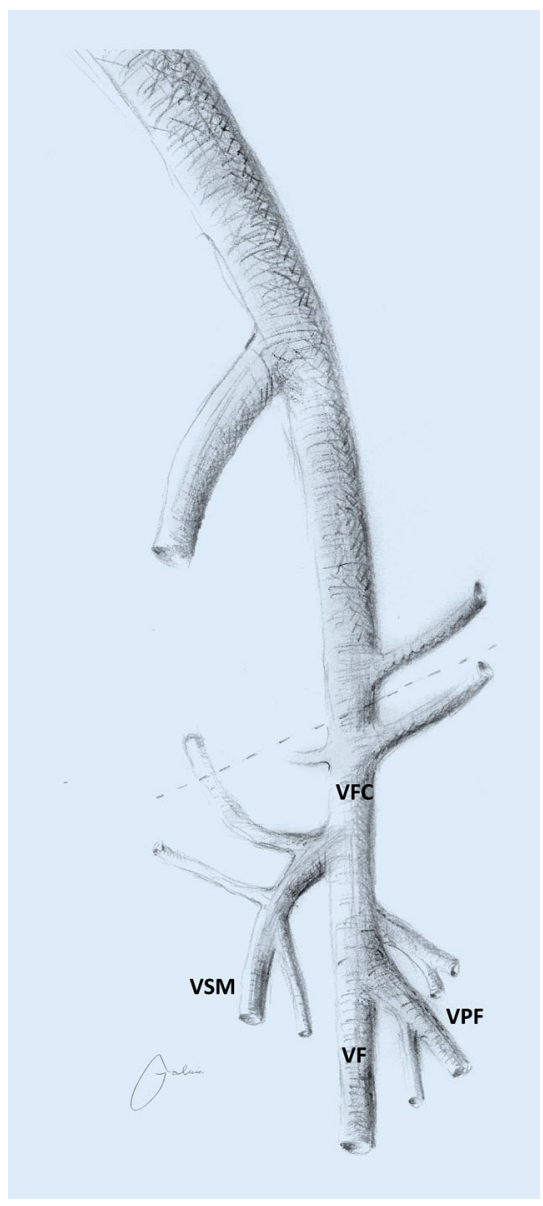

Fig. $1 \Delta$ Anatomy of the venous system in the inguinal region. VFC common femoral vein, VSM great saphenous vein $V F$ femoral vein, $V P F$ profunda femoris vein

prevents or reduces edema formation and improves microcirculation. Thus, compression therapy not only prevents but also improves PTS findings [4, 31]. In cases where compression therapy is contraindicated, such as severe peripheral arterial disease (PAD) and peripheral neuropathy, intermittent pneumatic compression therapy represents a reasonable alternative [38].

\section{Surgical treatment}

Palma and Esperon first described the surgical treatment of venous obstruction by means of a femorofemoral venous bypass using a saphenous vein crossover graft in 1959 [27]. Although this venous bypass achieves high long-term patency rates, venous obstruction is often inadequately treated due to the small diameter of the saphenous vein. For this

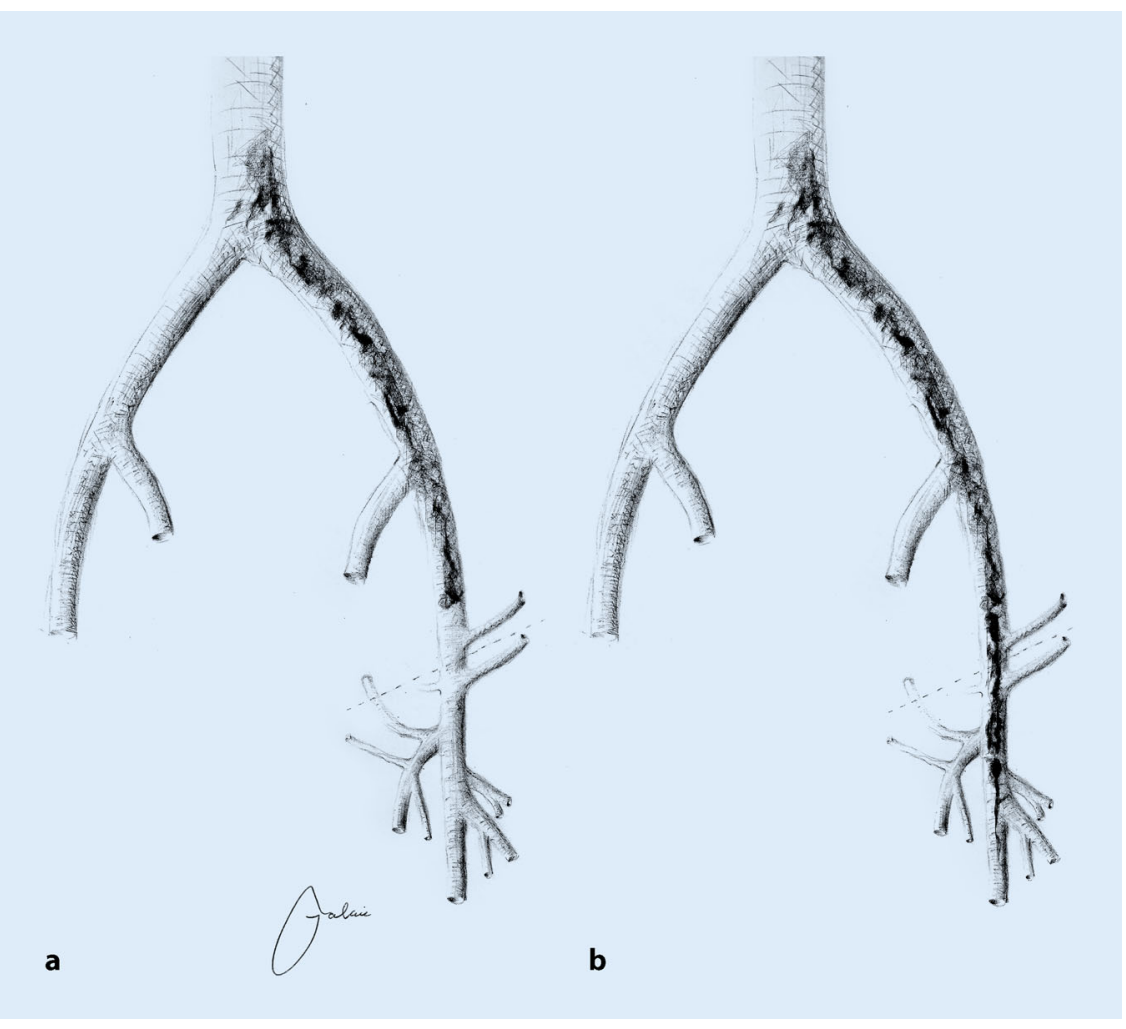

Fig. $2 \Delta$ Indications depending on the extent of chronic venous obstruction: a without common femoral vein involvement and $\mathbf{b}$ with common femoral vein involvement

reason, we favor large caliber, ring-reinforced polytetrafluoroethylene (PTFE) grafts with a diameter of $12-14 \mathrm{~mm}$.

\section{Interventional treatment}

While PTS significantly impairs patient quality of life, it is very rarely threatening to life or extremities; therefore, in our opinion, the objective should be to use as minimally invasive a treatment approach as possible. Percutaneous transfemoral recanalization of the iliac veins using stent angioplasty has become increasingly more important in recent years, ever since Neglén et al. first published the technique in 2000 [22]. It is now established as a low-risk treatment option with good long-term results.

\section{Indications}

The indications for recanalization in the presence of PTS need to be made with great caution. In the authors' opinion, interventional treatment is indicated in the case of significant impairment to the quality of life, particularly due to venous claudication or non-healing venous ulcers. Asymptomatic iliofemoral or iliocaval chronic venous obstruction (CVO) should be conservatively treated. Above all, complex iliocaval recanalization with or without endophlebectomy should only be performed in cases where the patient's level of suffering is high. Recanalization at the iliocaval level in the case of insufficient inflow from the periphery due to postthrombotic stenosis or occlusion of the femoral vein (FV) and deep femoral vein (DFV) that extends distally is likely to be ineffective and therefore contraindicated. Thus, the FV and DFV require careful preoperative evaluation (• Fig. 1).

In the case of inflow obstruction due to postthrombotic trabeculae in the region of the common femoral vein (CFV), endophlebectomy of this vein should be performed as part of interventional recanalization, possibly including creation of an arteriovenous (AV) fistula in order to ensure sufficient inflow and prevent early thrombotic occlusion $([40,15]$, - Fig. 2).

Patients need to be explicitly informed about the procedure, possible complica- 
tions, postoperative adjunctive therapy (e.g. anticoagulation and compression) and treatment results.

\section{Diagnostic work-up}

Using duplex ultrasound, combined with magnetic resonance (MR) phlebography or computed tomography (CT) phlebography, the extent of PTS can be visualized and surgery can be planned. The femoral and crural veins and venous valves in particular, as well as the morphology and function, can be well visualized using duplex ultrasound; however, MR and CT phlebography are better suited to the evaluation of iliocaval circulation. Both techniques are capable of achieving good visualization of stenosis, occlusion, atresia, external compression and collaterals. In patients with PTS we recommend performing gadolinium-enhanced MR phlebography, which additionally visualizes intraluminal trabeculae and vessel wall thickening; however, contrast medium administered for CT phlebography in the setting of PTS is unable to reach all vein segments, with the result that not all intravenous lesions can be detected and the extent of findings may be underestimated [2]. The extent of CVO needs to be differentiated preoperatively as follows:

1. Iliac or iliocaval CVO,

2. Iliac or iliocaval CVO with $\mathrm{CFV}$ involvement.

This classification is relevant for treatment as the former group can be treated using a purely endovascular approach but the latter possibly requires a hybrid procedure.

\section{Technical details of recanalization}

Irrespective of the location of obstruction, we perform recanalization with the patient in the supine position and under general anesthesia. Venous dilatation and stenting is painful and can take some time. Performing the procedure with the patient under local anesthesia should be reserved only for patients with circumscribed stenosis in the iliac region, e.g. May-Thurner syndrome. The CFV, FV, popliteal vein $(\mathrm{PV})$, right internal jugu-

Gefässchirurgie 2016 · [Suppl 2]: 21:S37-S44 DOI 10.1007/s00772-016-0156-4

(c) The Author(s) 2016 . This article is available at SpringerLink with Open Access

H. Jalaie · K. Schleimer · M. E. Barbati · A. Gombert · J. Grommes · M. A. F. de Wolf · R. de Graaf · C. H. A. Wittens

Interventional treatment of postthrombotic syndrome

\section{Abstract}

Background. Postthrombotic syndrome (PTS) is the development of symptoms and signs of chronic venous insufficiency following deep vein thrombosis (DVT) and has a significant negative effect on the quality of life. The current understanding is that the clinical manifestation of PTS is related more to venous obstruction than it is to venous reflux. The use of interventional techniques for the treatment of venous obstruction and/or venous occlusion has rapidly increased in recent years.

Objective. This article summarizes the current concept of endovenous and hybrid interventions and presents the optimized treatment of choice in patients with chronic symptomatic venous obstruction.

Methods. We performed a systematic literature search in the Medline database to identify relevant studies on the treatment of patients with PTS.
Results. A meta-analysis of the relevant studies showed that this minimally invasive procedure is an effective treatment option with low morbidity and no mortality. Use of the newly developed dedicated venous stents showed promising results with good midterm patency rates and a significant decrease in related symptoms.

Conclusion. Interventional therapy for the treatment of symptomatic chronic venous obstruction has become the method of choice in recent years. More studies are needed to evaluate the long-term success rate of dedicated venous stents.

Keywords

Postthrombotic syndrome - Chronic venous obstruction - Venous recanalization . Endophlebectomy · Venous stents

\section{Interventionelle Therapie des postthrombotischen Syndroms}

\section{Zusammenfassung}

Hintergrund. Das postthrombotische

Syndrom (PTS) als Langzeitfolge der tiefen Beinvenenthrombose (TVT) kann zu einer erheblichen Beeinträchtigung der Lebensqualität führen. Nach neuerem Erkenntnisstand trägt die obstruktive Komponente mehr zur Entstehung der Symptomatik bei als die refluxive. Interventionelle Verfahren zur Beseitigung der venösen Obstruktion/Okklusion haben im letzten Jahrzehnt rasant an Bedeutung zugenommen.

Zielsetzung. In diesem Beitrag wird die endovaskuläre bzw. Hybridbehandlung der symptomatischen chronisch-venösen Obstruktion und unser Konzept zur Optimierung derselben dargestellt.

Methoden. Die elektronische Datenbank "medline" wurde systematisch nach Artikeln in deutscher oder englischer Sprache durchsucht, welche die Behandlung des PTS thematisieren.
Ergebnisse. Alle zugrundeliegenden Studien zeigen, dass es sich bei dieser wenig invasiven Operation um eine effektive Behandlungsmethode ohne Mortalität mit wenig Morbidität handelt. Die Offenheitsraten sind gut. Es lässt sich ein signifikanter Rückgang der Beschwerden erzielen. Unsere Kurzzeitergebnisse mit speziellen venösen Stents sind erfolgversprechend.

Schlussfolgerung. Die interventionelle Therapie zur Behandlung der symptomatischen chronisch-venösen Obstruktion hat sich in den letzten Jahren zur Therapie der Wahl entwickelt. Die im Kurzzeitverlauf nachgewiesenen Vorteile der speziellen venösen Stents sollten anhand von kontrollierten Vergleichsstudien evaluiert werden.

\section{Schlüsselwörter}

Postthrombotisches Syndrom - Chronischvenöse Obstruktion - Venöse Rekanalisation . Endophlebektomie · Venöse Stents 

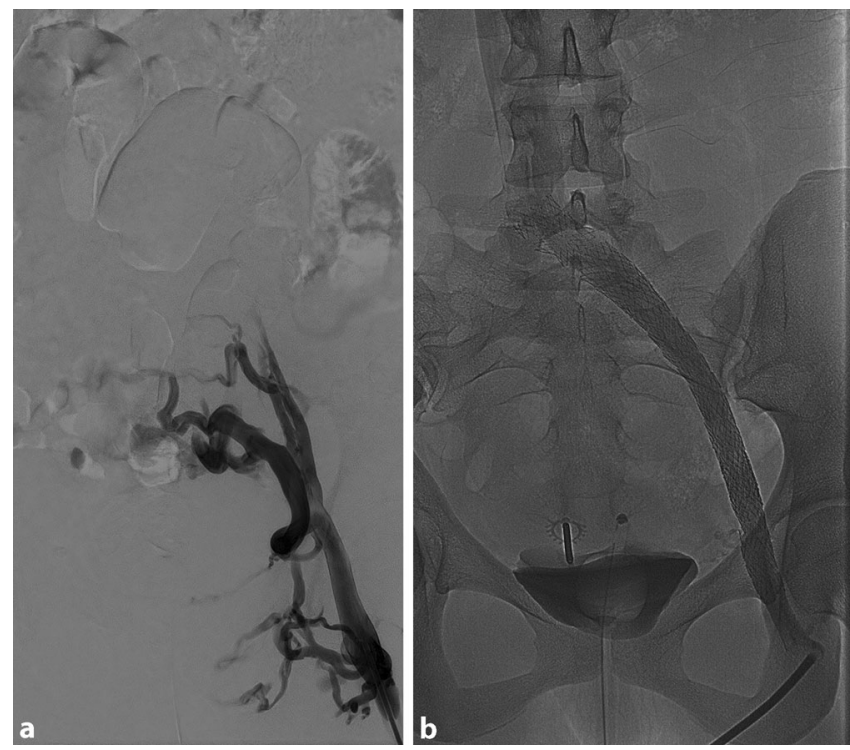

Fig. $3<$ Intraoperative phlebography a before recanalization and $\mathbf{b}$ after recanalization

lar vein and contralateral CFV are suitable for recanalization access. The great saphenous vein (GSV) and the DFV may be used for access as a last resort. In the case of CFV involvement, we favor the ipsilateral FV between the middle and proximal third as the puncture site. This enables the tip of the vascular sheath to be placed in front of the confluence of the DFV with the FV. In this way, the full length of the CFV can be assessed and the procedure performed according to the relevant pathology. Ultrasound-guided venous puncture is performed. Once a venous sheath has been introduced using the Seldinger technique, a variety of wires and catheters can be used to pass the stenosis or occlusion. For recanalization, we use a stiff hydrophilic wire supported by a slightly curved catheter. The recanalization of segments affected by postthrombotic changes can take between minutes and hours, depending on the extent of lesions. This requires a high level of patience and precision. Once the vein segment affected by postthrombotic changes has been passed, the hydrophilic wire should be exchanged for a more stable wire (super-stiff wire). The area of constriction or occlusion can then be dilated using a large lumen balloon. The vein should be predilated to at least the diameter of the planned stent. Postdilation is performed following stent placement. Stent-assisted percutaneous transluminal angioplasty (stent PTA) should be performed from healthy to healthy segments, i.e. the entire venous segment affected by postthrombotic changes should be repaired by stent PTA. Common iliac vein (CIV) constriction should always be stented over. At the same time, overly wide placement of the stent in the CIV should be avoided. Control phlebography in two planes is mandatory. Following successful recanalization, vigorous contrast medium outflow occurs along the stented iliac flow path. Collaterals should no longer be detectable ([9, 10]; • Fig. 3).

\section{Common femoral vein endophlebectomy and arteriovenous fistula creation during recanalization}

In the absence of sufficient inflow, early thrombotic occlusion is virtually unavoidable despite successful iliac stent PTA. The inflow is supplied primarily with venous blood from the FV, the DFV and the GSV. Even in the case of postthrombotic FV occlusion, collateral inflow via the DFV may be adequate. In the case of CFV involvement and hence also significantly impaired peripheral inflow, surgery should be performed as a hybrid procedure. The CFV is visualized and, following longitudinal venotomy, postthrombotic trabeculae are excised (๑ Fig. 4). Patch angioplasty is then performed using bovine patch material and in addition, an AV fistula is created between the common femoral artery and CFV using a ring-reinforced $6 \mathrm{~mm}$ PTFE graft in a loop configuration. Once peripheral venous inflow is ensured, stent PTA of the recanalized iliac or iliocaval vein segment is performed ([40, 15, 32]; - Fig. 5). As, here again, the basic principle is that the stent should terminate both proximally and distally in healthy vein segments, stent PTA up to the endophlebectomized CFV is necessary, i. e. the stent terminates, in contrast to recommendations in arterial PTA and stent placement, below the inguinal ligament.

\section{New venous stents}

Initially, the same stents as those used in arterial stent PTA (e.g. Wallstents and nitinol stents) were used for recanalization of the iliac venous circulation. Stent PTA of a vein affected by postthrombotic changes, intraluminal scarring and often also external compression cannot be compared with arterial stent PTA for arteriosclerosis; therefore, the stents used for venous recanalization have special requirements:

1 Vein diameter is greater than the diameter of the corresponding arteries. Stents with a diameter of $12-18 \mathrm{~mm}$ are used for venous iliac recanalization.

2 Postthrombotic lesions are generally extensive in length, making longer stents necessary. The deployment of several overlapping stents is inadequate to solve this problem, as this reduces the required flexibility.

3 Postthrombotic veins often show long segment scarring, in addition to which external pressure may be present, e.g. as in May-Thurner syndrome; therefore, stents with high radial strength are required.

4 In the case of venous recanalization, highly flexible stents that adapt to the anatomical course of the vein also during movement are required. The greatest angulation (up to $90^{\circ}$ ) while seated is seen particularly in the region of the iliac bifurcation, where the external iliac vein (EIV) crosses into the CIV (• Fig. 6). 

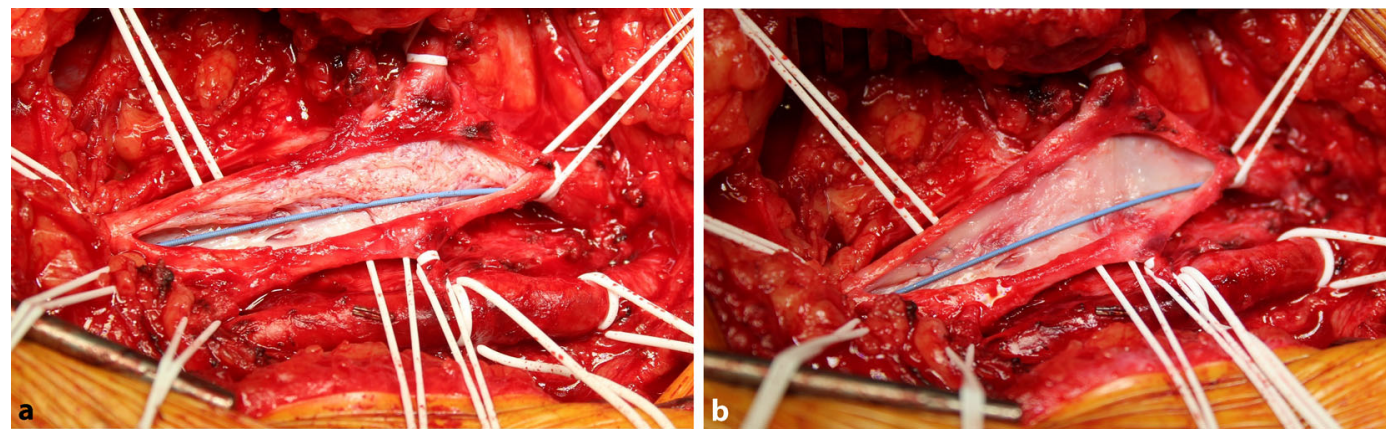

Fig. $4<$ Intraoperative site of common femoral vein endophlebectomy a once the wire has passed through the obstruction. Visualization of postthrombotic trabeculae $b$ following excision of postthrombotic trabeculae

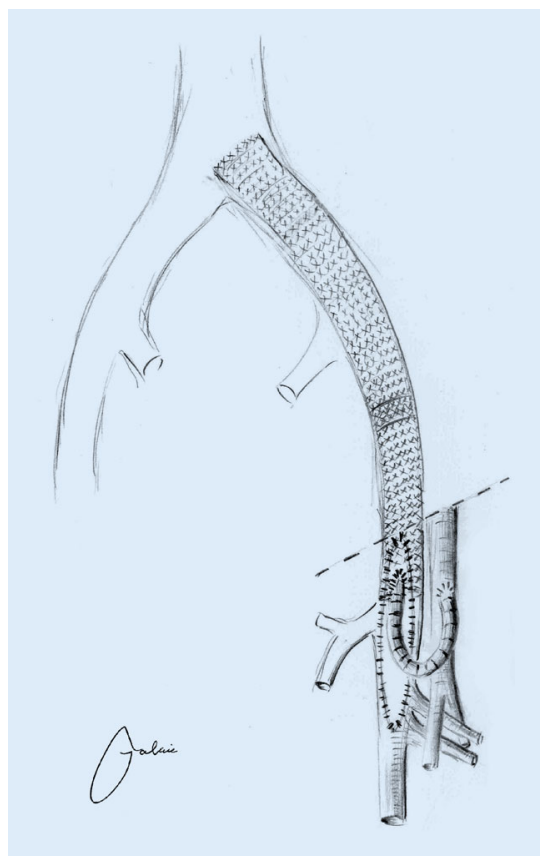

Fig. $5 \Delta$ Following recanalization of the pelvic vessels with endophlebectomy of the common femoral vein and patch angioplasty and placement of a loop-shaped arteriovenous fistula (ringed 6 mm PTFE)

This resulted in the development of special venous stents, such as the Veniti Vici (Veniti, St. Louis, MO), Zilver Vena (Cook, Bloomington, IN) and the sinus venous stent (OptiMed, Ettlingen, Germany). These dedicated venous stents combine high flexibility and strong radial force ([41]; • Fig. 7).

\section{Anticoagulation}

Following recanalization, coagulation is increased due to the intimal lesion resulting from endophlebectomy and stent placement. Furthermore, hematoma formation with compression of the revascularized vein segment as well as patient immobilization all play a role. The most

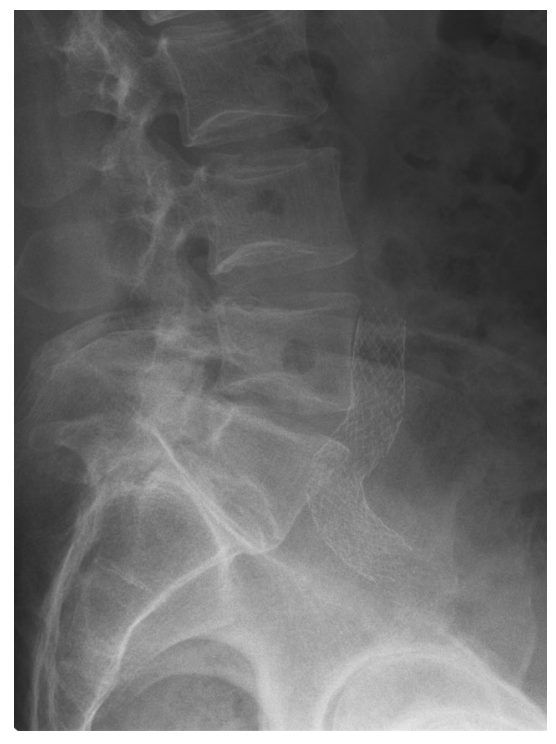

Fig. $6 \Delta$ Follow-up (lateral pelvic X-ray) following stent placement. Maximum flexibility ofavenous stent in the iliac bifurcation region

common postoperative complication is occlusion of the recanalized segment due to thrombosis. Thus, adequate perioperative and postoperative therapeutic anticoagulation are crucial. Therapeutic anticoagulation is not paused preoperatively and 5000 IU heparin is administered intraoperatively. Surgery is performed under regular monitoring of the activated clotting time (ACT), which should be $\geq 200$ s. Therapeutic anticoagulation is continued postoperatively. When using vitamin $\mathrm{K}$ antagonists for anticoagulation, the target International Normalized Ratio (INR) range should be 2.5-3.5. If values fall below the lower limit, we recommend additionally administering low-molecular heparin in a therapeutic dose. New oral anticoagulants (NOAC) are coming increasingly into use. At our clinic, therapeutic anticoagulation is continued for at least 6 months postoper- atively. Anticoagulation should be adjusted thereafter to the patient's individual risk of thrombosis recurrence [15].

\section{Postoperative adjunctive therapy}

Early mobilization is crucial to the success of interventional therapy. If this cannot be achieved, intermittent pneumatic compression therapy should be initiated. The wearing of compression stockings should be continued for at least 1 year postoperatively. A duplex ultrasound examination prior to discharge is recommended. We perform the first patient follow-up on the 14th postoperative day. Should thrombotic occlusion be detectable at that time, this can be adequately treated with local lysis. If stent-related complications are detected, these can be resolved following lysis via the same access.

\section{Current results using venous stents}

Between March 2012 and July 2014, we performed venous recanalization using sinus-Venous stents in 40 patients with PTS (excluding patients with compression syndrome and no PTS) at one of our centers. - Table 2 shows the demographic data of patients. Mean followup time was 5.5 months (range 1-18 months). There were no mortalities. The primary patency rate after 3, 6 and 12 months was $97 \%, 93 \%$ and $85 \%$, respectively. Rethrombosis was seen in three patients, which could be successfully treated using an endovascular approach. Stent-related complications are shown in - Table 3. Therapeutic anticoagulation caused minor bleeding not 

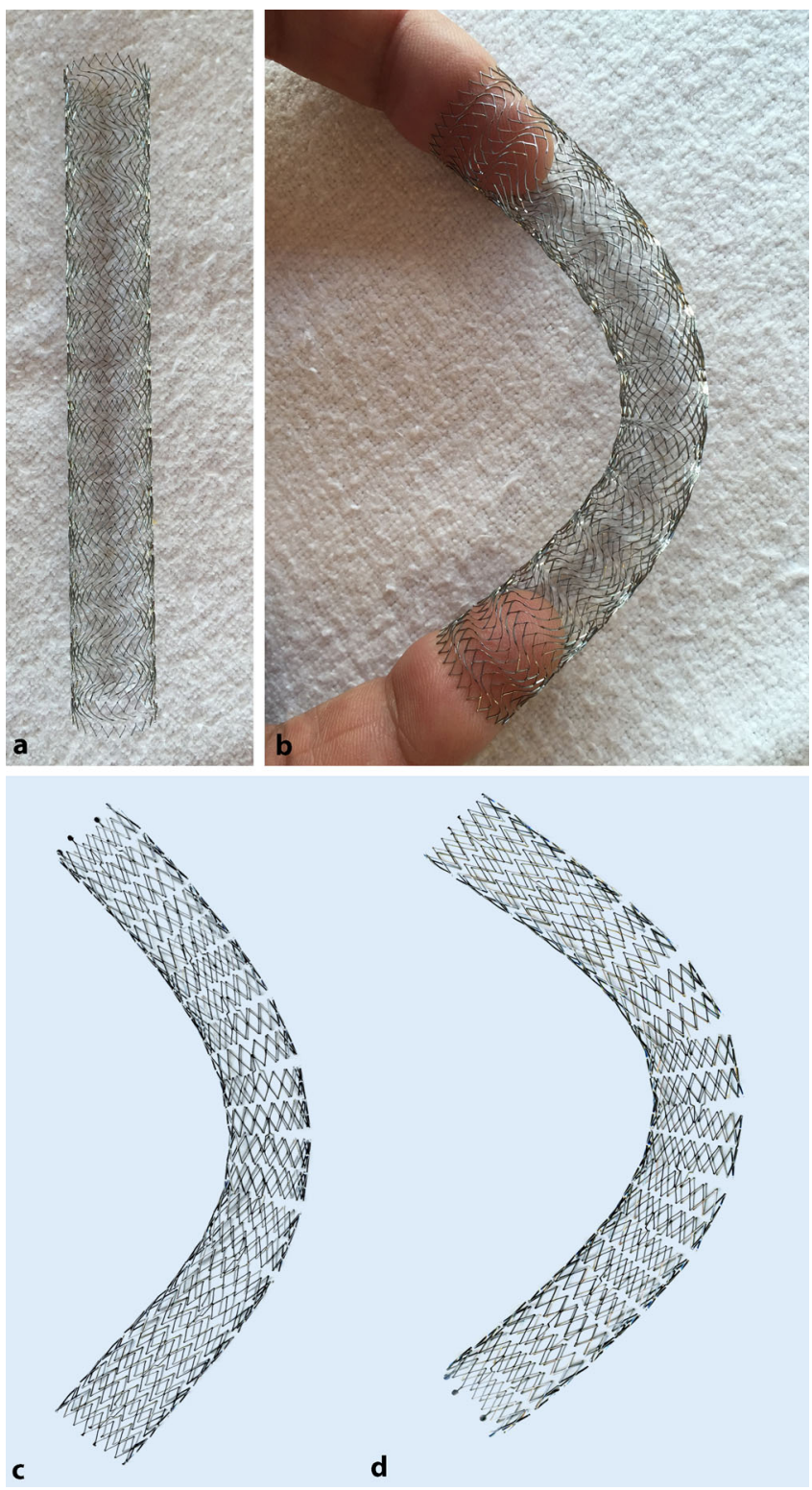

Fig. $7<\ln$ vitro demonstration of a venous stent. a, b Vici Venous Stent ${ }^{\circledast}$ (Veniti, St. Louis, MO), c, d Sinus-Venous stent (OptiMed, Ettlingen, Germany)

requiring treatment in five patients overall. The mean Villalta score decreased postoperatively from 11.5 (range 2-24) to 5.0 (range $0-16$ ) [41].

\section{Discussion}

Although PTS significantly impairs the quality of life, it rarely poses a threat to life or extremities. The goal of treatment is to alleviate symptoms and improve the quality of life. In our opinion this should be achieved with an approach that is as minimally invasive as possible. In 2000, Neglén et al. described the technique of interventional recanalization of the iliofemoral venous circulation [22] and published the largest study to date on the technique in 2006 ( $n=982$ patients and 464 with PTS). They primarily used Wallstents and, in a small number of cases, nitinol stents developed for the arterial system. Mortality was $0 \%$, while the primary patency rate was $57 \%$ in PTS patients after 72 months and the secondary patency rate was $86 \%$. Neglén et al. demonstrated a significant improvement in the quality of life in patients [23]. Hartung et al. published a study with 89 patients including 35 who suffered from PTS and performed stent PTA using only Wallstents. No cases of pulmonary embolism or mortality were observed perioperatively. The primary patency rates after 12 months, 3 years and 10 years were $89 \%, 83 \%$ and $83 \%$, respectively [11]. Blättler and Blättler [3], Raju et al. [34] and te Riele et al. published intermediate results (follow-up 9-15 months) showing primary patency rates of 58-79\% [37]. Compared with these intermediate results, our results using new venous stents are promising.

It is difficult to directly compare the available studies due to their inhomogeneous patient populations. The majority of studies mainly report about compression symdrome (e. g. May Thurner Syndrome), in which significantly better patency rates are achieved. A variety of stents were used, observational periods often varied significantly and while in some studies only interventional recanalization was performed, in others complex hybrid procedures were performed $[3,11$, 23, 24, 34, 37].

Interventional recanalization for venous femoro-iliocaval obstruction/ occlusion has proven to be a minimally invasive, safe and effective procedure. Convincing results are achieved with low morbidity, good patency rates, a significant reduction of symptoms and no mortality. We observed no symptom progression in the case of treatment failure and the option still remains to perform an open procedure.

\section{Conclusion}

Interventional venous recanalization is becoming increasingly more popular for the treatment of PTS. In order to ensure adequate results, the following aspects need to be taken into consideration:

1 Adequate inflow and optimal outflow in the stented vein segment are essential. This is achieved by performing stent PTA from healthy to healthy segments, early mobilization and compression therapy. Involvement of the CVF may necessitate endophlebectomy as well as creation of an AV fistula.

2 Only dedicated venous stents should be used.

3 Adequate perioperative and postoperative therapeutic anticoagulation is mandatory.

4 We recommend close follow-up. 
Table 2 Demographic data of patients with postthrombotic lesions

\begin{tabular}{|ll}
\hline Number & $\mathbf{4 0}(\%)$ \\
\hline Female & $24(53)$ \\
\hline Mean age (years) & $45(17-68)$ \\
\hline Isolated DVT & $28(70)$ \\
\hline Recurrent DVT & $7(18)$ \\
\hline Asymptomatic DVT & a \\
\hline $\begin{array}{l}\text { Time interval between DVT } \\
\text { and treatment (years, range) }\end{array}$ & $6(13)$ \\
\hline Left-sided DVT & $29(83)$ \\
\hline Right-sided DVT & $5(14)$ \\
\hline Bilateral DVT & $1(3)$ \\
\hline Moderate Villalta score & $11.5(2-24)$ \\
\hline Venous claudication & $25(63)$ \\
\hline Superficial venous reflux & $33(83)$ \\
\hline Deep venous reflux & $12(30)$ \\
\hline Perforating vein reflux & $5(13)$ \\
\hline
\end{tabular}

DVT deep vein thrombosis, PTS postthrombotic syndrome, CVO chronic venous obstruction

${ }^{a}$ Asymptomatic DVT: no history of DVT but clear symptoms/findings of PTS with CVO

Taking these premises into account, venous recanalization is an elegant and safe procedure associated with low morbidity and high patency rates.

\section{Corresponding address}

Dr. H. Jalaie
Klinik für Gefäßchirurgie,
Universitätsklinikum der
RWTH Aachen, Europäisches
Gefäßzentrum Aachen-
Maastricht
Pauwelstr. 30, 52074 Aachen,
Germany
hjalaie@ukaachen.de

\section{Compliance with ethical guidelines}

Conflict of interests. H. Jalaie, K. Schleimer, M. E. Barbati, A. Gombert, J. Grommes, M. A. F. de Wolf, R. de Graaf and C.H.A. Wittens state that there are no conflicts of interest.

The accompanying manuscript does not include studies on humans or animals.

The supplement containing this article is not sponsored by industry.

\begin{tabular}{|c|c|}
\hline Complications & $\begin{array}{l}\text { Patients } \\
(n=40, \%)\end{array}$ \\
\hline $\begin{array}{l}\text { Postoperative bleeding } \\
\text { (puncture site) }\end{array}$ & $1(2.5)$ \\
\hline $\begin{array}{l}\text { Readmission due to stent-re- } \\
\text { lated pain }\end{array}$ & $1(2.5)$ \\
\hline Persistent stent compression & $2(5)$ \\
\hline Stent tapering & $2(5)$ \\
\hline Stent stenosis & $2(5)$ \\
\hline $\begin{array}{l}\text { Insufficient stenting of the } \\
\text { area of compression }\end{array}$ & $2(5)$ \\
\hline
\end{tabular}

Open Access. This article is distributed under the terms of the Creative Commons Attribution 4.0 International License (http://creativecommons.org/ licenses/by/4.0/), which permits unrestricted use, distribution, and reproduction in any medium, provided you give appropriate credit to the original author(s) and the source, provide a link to the Creative Commons license, and indicate if changes were made.

\section{References}

1. Akesson H, Brudin L, Dahlstrom JA, Eklof B, Ohlin P, Plate $G$ (1990) Venous function assessed during a 5 year period after acute ilio-femoral venous thrombosis treated with anticoagulation. Eur J Vasc Surg 4(1):43-48

2. Arnoldussen $\mathrm{CW}$, Graaf $\mathrm{R}$ de, Wittens $\mathrm{CH}$, Haan MW de (2013) Value of magnetic resonance venography and computed tomographic venography in lower extremity chronic venous disease. Phlebology 28(Suppl 1):169-175. doi:10.1177/0268355513477785

3. Blättler W, Blättler IK (1999) Relilef of obstructive pelvic venous symptoms with endoluminal stenting. J Vasc Surg 29(3):484-488

4. Brandjes DP, Buller HR, Heijboer H, Huisman MV, Rijk M de, Jagt $\mathrm{H}$ et al (1997) Randomised trial of effect of compression stockings in patients with symptomatic proximal-vein thrombosis. Lancet 349(9054):759-762. doi:10.1016/s01406736(96)12215-7

5. Chen JT, Comerota AJ (2012) Detecting the association between residual thrombus and post-thrombotic classification of chronic venous disease with range regression. Rev Recent Clin Trials 7(4):329-334

6. Cogo A, Lensing AW, Prandoni P, Hirsh J (1993) Distribution of thrombosis in patients with symptomatic deep vein thrombosis. Implications for simplifying the diagnostic process with compression ultrasound. Arch Intern Med 153(24):2777-2780

7. Comerota AJ, Grewal N, Martinez JT, Chen JT, Disalle $\mathrm{R}$, Andrews Letal (2012) Postthrombotic morbidity correlates with residual thrombus following catheter-directed thrombolysis for iliofemoral deep vein thrombosis. J Vasc Surg 55(3):768-773. doi:10.1016/j.jvs.2011.10.032

8. Deroo S, Deatrick KB, Henke PK (2010) The vessel wall: $A$ forgotten player in post thrombotic syndrome. Thromb Haemost 104(4):681-692. doi:10.1160/th10-03-0183.
9. Graaf R de, Arnoldussen C, Wittens CH (2013) Stenting for chronic venous obstructions a new era. Phlebology 28(Suppl 1):117-122. doi:10.1177/0268355513476449

10. Graaf R de, Wolf M de, Sailer AM, Laanen J van, Wittens C, Jalaie H (2015) Iliocaval confluence stenting for chronic venous obstructions. Cardiovasc Intervent Radiol 2015(03):17-25772400. doi:10.1007/s00270-015-1068-5

11. Hartung $O$, Loundou AD, Barthelemy $P$, Arnoux D, Boufi M, Alimi YS (2009) Endovascular management of chronic disabling ilio-caval obstructive lesions: long-term results. Eur JVasc Endovasc Surg 38(1):118-124. doi:10.1016/j.ejvs.2009.03.004.

12. Henke PK, Comerota AJ (2011) An update on etiology, prevention, and therapy of postthrombotic syndrome. J Vasc Surg 53(2):500-509. doi:10.1016/j.jvs.2010.08.050.

13. Henke PK, Wakefield T (2009) Thrombus resolution and vein wall injury: dependence on chemokines and leukocytes. Thromb Res 123(Suppl 4):S72-S78. doi:10.1016/s00493848(09)70148-3.

14. Hull RD, Marder VJ, Mah AF, Biel RK, Brant RF (2005) Quantitative assessment of thrombus burden predicts the outcome of treatment for venous thrombosis: a systematic review. Am J Med 118(5):456-464 doi:10.1016/j.amjmed.2005.01.025.

15. Jalaie $H$, Arnoldussen $C$, Barbati $M$, Kurstjens R, Graaf R de, Grommes J et al (2014) What predicts outcome after recanalization of chronic venous obstruction: hemodynamic factors, stent geometry, patient selection, anticoagulation or other factors? Phlebology 29(1 suppl):97-103. doi:10.1177/0268355514529510.

16. Kahn SR, Ginsberg JS (2002) The post-thrombotic syndrome: current knowledge, controversies, and directions for future research. Blood Rev 16(3):155-165

17. Kahn SR, Ginsberg JS (2004) Relationship between deep venous thrombosis and the postthrombotic syndrome. Arch Intern Med 164(1):17-26. doi:10.1001/archinte.164.1.17.

18. Kahn SR, Hirsch A, Shrier I (2002) Effect of postthrombotic syndrome on health-related quality of life after deep venous thrombosis. Arch Intern Med 162(10):1144-1148

19. Kahn SR, Shbaklo H, Lamping DL, Holcroft CA, Shrier I, Miron MJ, Roussin A, Desmarais S, Joyal F, Kassis J, Solymoss S, Desjardins L, Johri M, Ginsberg JS (2008) Determinants of health-related quality of life during the 2 years following deep vein thrombosis. J Thromb Haemost 6(7):1105-1112. doi:10.1111/j.1538-7836.2008.03002.x.

20. Labropoulos N, Volteas N, Leon M, Sowade O, Rulo A, Giannoukas AD et al (1997) The role of venous outflow obstruction in patients with chronic venous dysfunction. Arch Surg 132(1):46-51

21. Launois R, Reboul-Marty J, Henry B (1996) Construction and validation of a quality of life questionnaire in chronic lower limb venous insufficiency (CIVIQ). Qual Life Res 5(6):539-554

22. Neglén P, Berry MA, Raju S (2000) Endovascular surgery in the treatment of chronic primary and post-thrombotic iliac vein obstruction. Eur J Vasc Endovasc Surg 20(6):560-571. doi:10.1053/ ejvs.2000.1251.

23. Neglén P, Hollis KC, Olivier J, Raju S (2007) Stenting of the venous outflow in chronic venous disease: long-term stent-related outcome, clinical, and hemodynamic result. J Vasc Surg 46(5):979-990. doi:10.1016/j.jvs.2007.06.046. 


\section{Leitthema}

24. Neglén P, Tackett TP, Raju S (2008) Venous stenting across the inguinal ligament. J Vasc Surg 48(5):1255-1261. doi:10.1016/j.jvs.2008.06.035.

25. O'Donnell TF Jr., Browse NL, Burnand KG, Thomas $M L$ (1977) The socioeconomic effects of an iliofemoral venous thrombosis. J Surg Res 22(5):483-488

26. O'Sullivan GJ, Sheehan J, Lohan D, McCann-Brown JA (2013) Iliofemoral venous stenting extending into the femoral region: initial clinical experience with the purpose-designed Zilver Vena stent. JCardiovasc Surg (Torino) 54(2):255-261

27. Palma EC, Esperon R (1959) Treatment of postthrombophlebitic syndrome by transplantation of the internal saphenous. Angiologia 11(2):87-94

28. Phillips LJ 2nd, Sarkar R (2007) Molecular characterization of post-thrombotic syndrome. J Vasc Surg 45(Suppl A):A116-A122. doi:10.1016/j.jvs.2007.02.034.

29. Plate $G$, Akesson $H$, Einarsson $E$, Ohlin P, Eklof $B$ (1990) Long-term results of venous thrombectomy combined with a temporary arterio-venous fistula. Eur JVasc Surg 4(5):483-489

30. Prandoni P, Lensing AW, Cogo A, Cuppini S, Villalta S, Carta Met al (1996) The long-term clinical course of acute deep venous thrombosis. Ann Intern Med 125(1):1-7

31. Prandoni $P$, Lensing AW, Prins $M H$, Frulla $M$, Marchiori A, Bernardi E et al (2004) Below-knee elastic compression stockings to prevent the postthrombotic syndrome: a randomized, controlled trial. Ann Intern Med 141(4):249-256

32. Puggioni A, Kistner RL, Eklof B, Lurie $F$ (2004) Surgical disobliteration of postthrombotic deep veins - endophlebectomy - is feasible. J Vasc Surg 39(5):1048-1052 (discussion 52) doi:10.1016/j.jvs.2003.12.036

33. Qvarfordt P, Eklof B, Ohlin P (1983) Intramuscular pressure in the lower leg in deep vein thrombosis and phlegmasia cerulae dolens. Ann Surg 197(4):450-453

34. Raju S, Hollis K, Neglen P (2006) Obstructive lesions of the inferior vena cava: clinical features and endovenous treatment. JVasc Surg 44(4):820-827

35. Singh H, Masuda EM (2005) Comparing short-term outcomes of femoral-popliteal and iliofemoral deep venous thrombosis: early lysis and development of reflux. Ann Vasc Surg 19(1):74-79. doi:10.1007/s10016-004-0133-3.

36. Strijkers RH, Wittens CH, Kahn SR (2012) Villalta scale: goals and limitations. Phlebology 27(Suppl 1):130-135. doi:10.1258/phleb.2011.012S02.

37. Riele WW te, Overtoom TT, Berg JC van den, PavoordtEDvan de, VriesJPde (2006) Endovascular recanalization of chronic long-segment occlusions of the inferior vena cava: midterm results. JEndovasc Ther 13(2):249-253

38. Van der Velden SK, Neumann HAM (2014) Compression in DVT treatment. Phlebology 29(5):331 doi:10.1177/0268355514537430

39. White RH (2003) The epidemiology of venous thromboembolism. Circulation 107:14-8. doi:10.1161/01.cir.0000078468.11849.66.

40. Wolf MA de, Arnoldussen CW, Wittens CH (2013) Indications for endophlebectomy and/or arteriovenous fistula after stenting. Phlebology 28(Suppl 1):123-128. doi:10.1177/0268355513477063.

41. Wolf MA de, Graaf $R$ de, Kurstjens RL, Penninx S, Jalaie H, Wittens CH (2015) Short-Term Clinical Experience with a Dedicated Venous Nitinol Stent: Initial Results with the SinusVenous Stent. Eur J Vasc Endovasc Surg: doi:10.1016/j.ejvs.2015.05.011. 